\title{
L'objet monde au Moyen Âge : clôtures et mise en ordre dans les productions littéraires, iconographiques et architecturales
}

Journée d'études, Paris, 10 février 2020

Mathieu Beaud, Luce Carteron, Kristina Mitalaïte et Enimie Rouquette

\section{(2) OpenEdition}

\section{Journals}

Édition électronique

URL : https://journals.openedition.org/cem/17932

DOI : 10.4000/cem. 17932

ISSN : 1954-3093

\section{Éditeur}

Centre d'études médiévales Saint-Germain d'Auxerre

Référence électronique

Mathieu Beaud, Luce Carteron, Kristina Mitalaïte et Enimie Rouquette, «L'objet monde au Moyen Âge : clôtures et mise en ordre dans les productions littéraires, iconographiques et architecturales ", Bulletin du centre d'études médiévales d'Auxerre | BUCEMA [En ligne], 24.2 | 2020, mis en ligne le 19 décembre 2020, consulté le 22 septembre 2022. URL : http://journals.openedition.org/cem/17932 ; DOI : https:// doi.org/10.4000/cem. 17932

Ce document a été généré automatiquement le 22 septembre 2022.

Creative Commons - Attribution - Pas d'Utilisation Commerciale - Partage dans les Mêmes Conditions 4.0 International - CC BY-NC-SA 4.0

https://creativecommons.org/licenses/by-nc-sa/4.0/ 
L'objet monde au Moyen Âge : clôtures et mise en ordre dans les productions littéraires, iconographiques et architecturales

Journée d'études, Paris, 10 février 2020

Mathieu Beaud, Luce Carteron, Kristina Mitalaïte et Enimie Rouquette

Introduction 
1 Cette rencontre, organisée le 10 février 2020 à la Maison de la Recherche Sorbonne-Nouvelle ${ }^{1}$, visait à questionner la notion de clôture comme mode de conception, de lecture et d'analyse des productions humaines médiévales envisagées dans leur singularité. Six universitaires de diverses disciplines $y$ présentèrent une œuvre par ce prisme d'interrogation. L'attente était de cerner et d'individualiser les concepts de clôture et de création, autant que possible dans leur formulation médiévale, et de les circonscrire sur les plans chronologique et contextuel. Cet article est la synthèse problématisée des communications, des discussions et des apports de la table ronde qui conclut la journée.

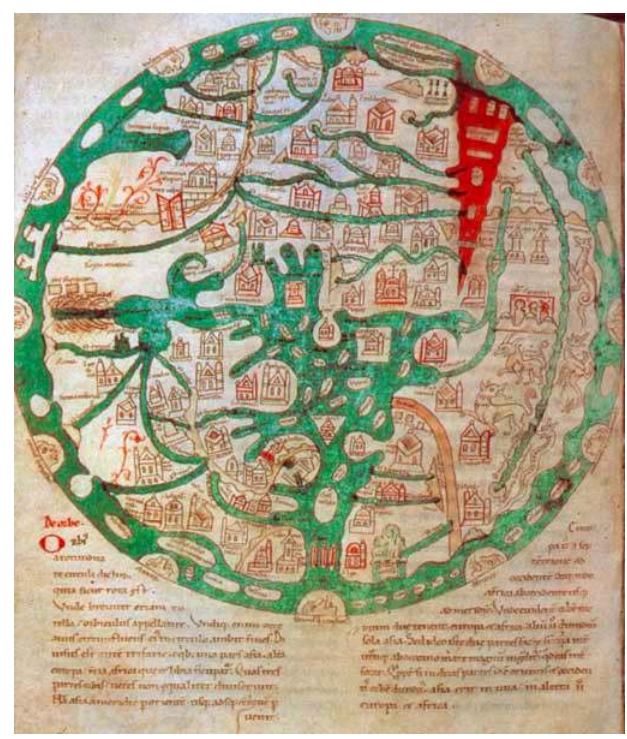

2 La rencontre s'est articulée autour d'« objets " médiévaux - entendus comme toute production posée, selon l'étymologie du terme objectum, face à la vue et à l'esprit de qui peut l'interpréter - pour les soumettre à une réflexion adoptant le concept de clôture comme principe de leur production.

3 Tout objet, en effet, en tant qu'il est achevé, donc clos, constitue un ensemble et une unité de sens. La correspondance analogique entre microcosme et macrocosme conçoit l'homme comme un "monde miniature" (homo minor mundus)². Cette idée, déjà présente dans le platonisme puis le néoplatonisme, se complexifie et se christianise dans le creuset patristique. C'est ainsi que l'homme " microcosme » apparait dans les écrits d'Isidore de Séville et de Bède le Vénérable ${ }^{3}$. Ce paradigme invite légitimement à interroger l'analogie entre la production humaine et la création divine. Les productions médiévales, souvent élaborées par correspondances proportionnelles entre macrocosme et microcosme, sont conçues de façon modulaire comme une répétition du tout dans ses parties ${ }^{4}$.

4 Or, puisque le monde est considéré comme une entité à la fois ordonnée et parfaite - ce que révèle par exemple la définition par Isidore du mundus, rapproché du mot grec kosmos $^{5}$ - et que sa perfection procède de son intégrité - toute la production de Dieu est dans le monde, il n'y a rien hors du monde si ce n'est Dieu (Ps 138, 7-12) -, il a ainsi semblé qu'en vertu de ce principe de correspondance, les productions humaines devaient également être envisagées sous l'angle de la clôture qui les achève.

5 L'époque carolingienne offrait un point de départ propice pour interroger la notion de clôture, par exemple par sa réflexion sur l'idéal monastique - en témoignent les réformes de Benoît d'Aniane ou encore le plan du monastère de Saint-Gall. La séparation entre le moine et le siècle s'accentue, et avec elle la délimitation d'un espace clos et autonome formé par le monastère, image du monde paradisiaque. Le De Laudibus sanctae crucis de Raban Maur constitue un autre exemple de figuration du monde, à l'échelle d'un texte: en agençant, en un seul dispositif, écriture, graphie et visuel, le lecteur/regardeur est invité à une contemplation, une méditation, une intellection potentiellement infinies et pourtant fondées sur les éléments que contient la page. On 
peut enfin penser au poème de Théodulf d'Orléans décrivant un arbre de la philosophie, dont les branches portent les sept arts libéraux et les vertus cardinales, inscrit dans un disque - Discus erat tereti formatus imagine mundi ${ }^{6}$ : « Il y avait un disque de forme ronde, à l'image du monde. » Le poème révèle une aspiration à un encyclopédisme hérité de l'Antiquité conçu comme un savoir couvrant tous les aspects du mundus, caractérisé par l'unité et la clôture, que symbolise le disque. Ainsi, nombre de productions carolingiennes proposent à leur échelle une reproduction du monde que l'humain peut embrasser de ses sens ou par l'intellect.

6 Parce qu'elle engendre du sens à partir des éléments qu'elle contient et de leur organisation, la clôture initie un processus dynamique qui, en recréant le monde à l'échelle d'un objet en une mise en ordre significative, en donne une version cohérente. Pour vérifier cette hypothèse, la rencontre interrogeait différents types d'objets rarement comparés, réunis selon trois façons d'approcher le mundus dans sa totalité : le schématiser, le récréer et le modéliser.

\section{Schématiser le monde}

7 La correspondance entre le monde comme création divine et la production humaine comme monde mène à considérer certaines de ces productions comme un aperçu du monde dans sa totalité. C'est le cas des mappemondes, schémas ou autres constructions diagrammatiques.

8 Emmanuelle Vagnon ouvre la journée par une réflexion sur la mappemonde (fig. 1) accompagnant une copie des Étymologies d'Isidore de Séville que conserve un manuscrit de la Bayerische Staatsbibliothek de Munich ( $\operatorname{clm} 10058$, fol. $154 v^{\circ}$, ca 1130) ${ }^{7}$. 
Fig. 1. Mappemonde de Munich, MÜNCHEN, Bayerische Staatsbibliothek, clm 10058, fol. $154 v^{\circ}$ (cl. Bayerische Staatsbibliothek).

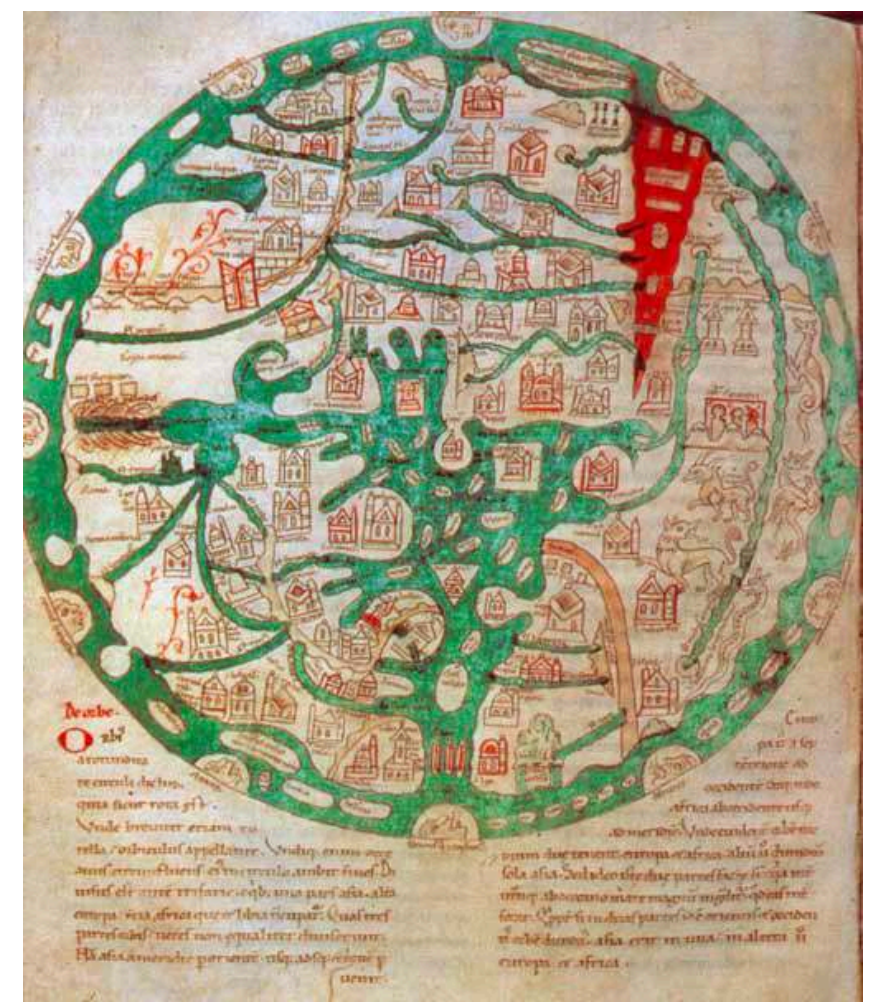

Les descriptions du monde du XII siècle sont majoritairement fondées sur celle d'Isidore, mais la mappemonde de Munich dépasse en richesse le contenu du texte qu'elle accompagne. Elle est à rapprocher de la Descriptio mappae mundi d'Hugues de Saint-Victor (1096-1141) conçue pour enseigner ce savoir encyclopédique à partir d'une mappemonde figurée, monumentale, dont celle du manuscrit serait une version simplifiée ${ }^{8}$.

10 La mappemonde de Munich rappelle ainsi le rôle pédagogique de l'image dans l'école victorine pour conduire les élèves des realia du monde à la compréhension de la Loi invisible qui le régit. Chaque lieu y est signalé - par des pictogrammes, emblèmes ou légendes - et disposé sur des surfaces délimitées par des moyens graphiques (lignes, couleurs, dessins) et beaucoup plus détaillées que la simple structure en T-O des cartes tardo-antiques. Enfin, la mappemonde est cerclée d'un océan ponctué des personnifications des vents soufflant vers le centre. Le monde habité (l'œkoumène) est ainsi disposé en un discours cohérent sur un disque orienté, sectorisé et clos.

11 Le De arca Noe d'Hugues fonctionne selon des modalités semblables en invitant le lecteur (ou l'auditeur) à reconstruire mentalement l'arche de Noé en une image allégorique, en trois dimensions, qui associe l'arche, l'âme humaine et le cosmos en tant que trois projections d'une même harmonie des divines proportions dictées à Noé. Même si certaines tentatives de reconstitutions ont été opérées, l'objet mental ainsi produit est en définitive irreprésentable; le discours qui le décrit devait servir de support à l'exercice intellectuel en disposant les choses à mémoriser dans des lieux imaginaires. Dans l'arche se trouve une mappemonde intellectuelle. Alors que la mappemonde du manuscrit de Munich est fondée sur des realia géographiques peu christianisés - elle n'est pas centrée sur Jérusalem, par exemple -, la description de 
l'arche emploie les noms de lieux et les points cardinaux dans un sens délibérément allégorique, pour mettre en discours le parcours historique de la christianisation.

Les principes victorins de modélisation cosmographique ressortent de la comparaison de ces documents. La description (descriptio) liste les choses contenues dans un ensemble. La discretio les distingue et les singularise dans leur lieu propre. Le duo descriptio/discretio favorise certes la mémorisation, l'enseignement et la transmission des savoirs (artes memoriae), mais invite aussi à une procession intellectuelle (deambulatio) qui amorce le processus méditatif. Ces trois principes de la théologie de la contemplation au XII siècle visent à résumer et à circonscrire la Création par l'image, mentale ou graphique, conçue autant comme une liste organisée du savoir encyclopédique qu'une allégorie cosmographique et concourent au saisissement de la totalité du monde pour approcher la compréhension de la loi divine qui le régit.

Thomas Le Gouge apporte un contrepoint à l'intervention précédente en analysant un diagramme dessiné et commenté par Albert le Grand au début des années 1250 dans le De causis proprietatum elementorum (fig. 2$)^{9}$, traité attribué au XIII ${ }^{\mathrm{e}}$ siècle à Aristote - en réalité écrit par un auteur arabe au $\mathrm{IX}^{\mathrm{e}}$ siècle ${ }^{10}$.

Fig. 2. ALBERT LE GRAND, De causis, WIEN, Österreichische Nationalbibliothek, cod. 273 , fol. $158 v^{\circ}$ (cl. T. Le Gouge).

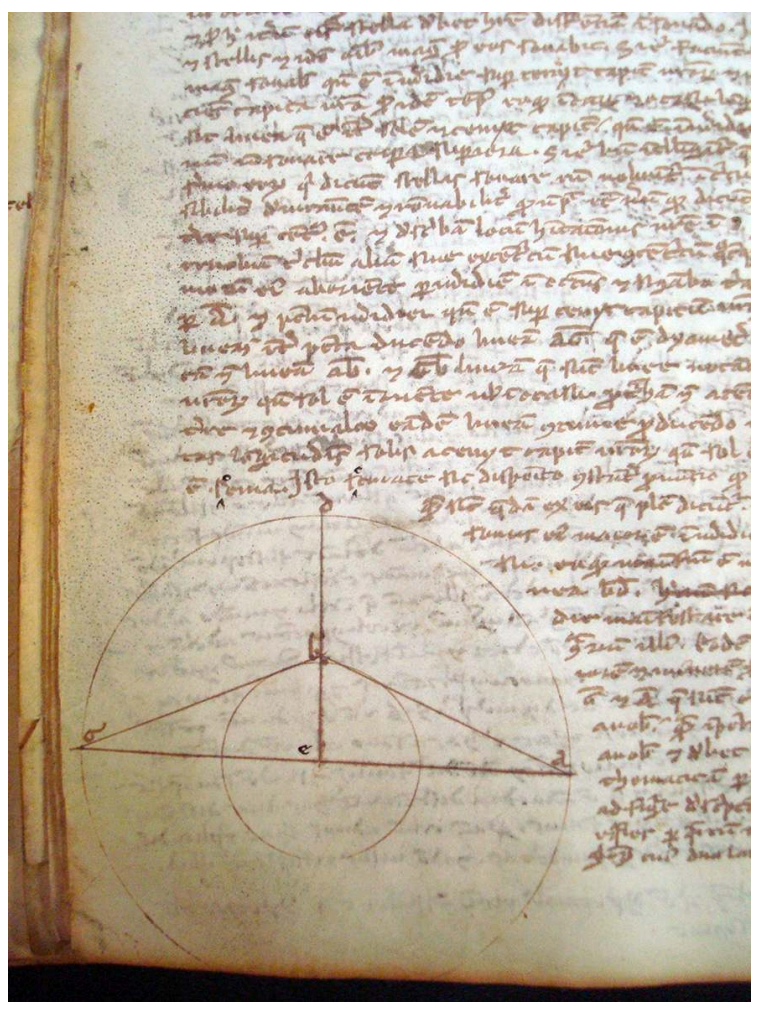

Le même diagramme a été réutilisé et commenté dans un ensemble de manuscrits glosés du De sphaera de Johannes de Sacrobosco ${ }^{11}$. La démarche de ces deux auteurs s'inscrit dans un changement de paradigme qui s'impose progressivement au $\mathrm{XIII}^{\mathrm{e}}$ siècle : à cette époque, en effet, l'expérience empirique s'associe à une abstraction géométrique nouvelle, qui s'appuie sur Euclide, et contribue à façonner ce que l'intervenant appelle l'image de la sphère. 
15 Dans le De causis, pour démontrer que le ciel ne joue pas de musique, contre ce qu'affirment les auteurs néoplatoniciens et des pythagoriciens (les physiologi), Albert commente en détail le schéma qui se trouve au début du livre II, et qu'il reproduit (ou fait reproduire) sur le manuscrit autographe du traité conservé à Vienne ${ }^{12}$. Pour Albert le Grand, si le ciel émettait de la musique, ce son serait nécessairement variable en raison des distances changeantes entre les corps célestes et les corps humains diversement positionnés sur la sphère terrestre. Cette variation d'intensité serait de fait perceptible par l'oreille humaine. Le schéma étudié matérialise ainsi l'idée que le son produit par le soleil devrait être plus fort à midi que le matin et le soir, la mesure du segment reliant une position sur la terre au soleil étant fluctuante. Par ailleurs, si ce son émis par les sphères devait s'estomper devant l'incommensurabilité du cosmos raison de son inaudibilité selon les physiologi -, Albert insiste sur le fait que l'argument vaut pour la ceinture zodiacale, mais pas pour les orbes des planètes.

16 Dans un groupe de manuscrits glosés du De sphaera de Sacrobosco, datés des années 1270, le même schéma réapparaît. Or, cette fois-ci, il s'agit de démontrer que la terre est au centre de l'univers, car elle est si petite par rapport au ciel que, peu importe où l'on se trouve sur la terre, quand on regarde vers les limites de l'univers, on voit toujours la même chose : l'horizon, qui sépare en deux moitiés égales la terre et le ciel. Le schéma, bien que similaire, prend alors un caractère très différent, puisque le cercle extérieur ne désigne plus l'orbe d'une planète mais la sphère de l'univers, la distance entre la terre et le ciel n'étant plus relative à la position d'un astre, mais incommensurable.

D’Albert le Grand aux manuscrits du De sphaera des années 1270, nous observons donc deux étapes dans l'avènement d'un nouveau paradigme, au cours duquel est opérée une mise en abstraction géométrique d'un cosmos incommensurable à la fois clos et infini.

\section{Recréer le monde}

Deux communications ont envisagé d'autres types de production, qui visent moins à rendre compréhensible le monde qu'à en reproduire une version par la parole ou par l'image.

19 En 2018, Maud Pérez-Simon a, avec son équipe, observé, photographié et étudié la charpente peinte vers 1450 de l'église sicilienne San Nicolo de Nicosi. Elle a présenté ses premières observations lors de la rencontre, tandis que les clichés des peintures étaient exposés à la Maison de la Recherche - Sorbonne-Nouvelle ${ }^{13}$.

Le plafond, découvert il y a trente ans sous la voûte actuelle ${ }^{14}$, comprend quatorze travées. En dépit des manques, une analyse iconographique d'ensemble est possible. L'intervenante interroge d'abord la définition de «monde», pouvant désigner l'« ensemble des choses créées », le "globe terrestre », la «façon dont les hommes l'occupent " ou le "domaine profane ", pour finalement distinguer trois paramètres pouvant faire de l'ensemble peint un monde : l'exhaustivité, l'ordre et la clôture.

21 Les peintres ont d'abord voulu condenser la variété de la Création et de la société. La diversité des motifs fait de ce plafond un monde imprévisible montré dans sa complexité et son exubérance.

22 Cependant, une structure apparaît si l'on superpose le sens d'occupation du plafond et l'orientation ouest-est de l'édifice. Se trouve par exemple à l'est un alphabet entier. Si celui-ci jouait un rôle dans le rituel de consécration des églises, le regroupement des 
lettres par quatre ainsi que leur ornementation évoquent davantage les techniques d'apprentissage en six jours de l'alphabet, qui était moralisé. Le Verbe surplombe ainsi en Orient la Création, alors qu'en Occident lui répond un personnage pénitent porteur de la sphère du monde, placé là comme un rappel de la correspondance entre microcosme et macrocosme.

Ce monde peint au plafond aurait ainsi deux extrémités, entre lesquelles se déploie un monde terrestre compartimenté et ordonné. L'axe longitudinal de l'église est marqué par une procession de saints disposés au milieu de chaque travée. Le clergé, tuteur de la société, tolère le désordre alentour parce que l'Église parvient à le circonscrire. Si cet axe de sainteté ne semble pas réellement organisé, une seconde structure d'ensemble, centralisée, apparaît : le spiritus se trouve au milieu, et la chair à l'entour.

L'intervenante établit pour conclure un lien avec le plafond (remanié) de l'église suisse Saint-Martin de Zillis (XII ${ }^{\mathrm{e}}$ siècle), où l'histoire évangélique, le spiritus, centralise et organise le mundus jusqu'à sa marginalité monstrueuse. Durant les trois siècles qui séparent les deux plafonds, le spiritus central s'est réduit au profit d'un mundus complexifié, mais la clôture est restée garante de la totalité du monde, en le formant et en le fermant, sans toutefois agir sur son organisation interne.

Marie-Céline Isaïa historicise la réflexion par l'analyse de Vitae ${ }^{15}$. L'emploi de mundus pour qualifier le monde matériel est d'abord dépréciatif dans l'hagiographie médiolatine : le saint doit s'en extraire $(\mathrm{Jn} 16,33)$ ou y combattre la tentation. En qualifiant progressivement ce monde périssable de saeculum, les hagiographes autorisent cependant l'emploi positif de mundus pour désigner la Création. Le terme implique alors des limites et un dessein puisque l'Église a pour mission de dominer « de la mer à la mer et du fleuve jusqu'aux limites de l'orbe terrestre » (Ps 71, 8, voir ex. 1). Parler de mundus revêt donc une dimension performative : l'Église et les saints ont à réaliser la perfection du mundus par l'annonce de l'Évangile. Grégoire de Tours créé le modèle herméneutique le plus influent sur ce sujet avec sa préface cosmique de la Vita patrum (ex. 2).

26 À partir des années 750-780, des hagiographes accentuent le caractère historique de cette lecture cosmique en situant la vie du saint local dans la continuité de l'histoire sainte - préface en postquam, ex. 3. Le saint est destiné par la providence à arriver en un temps donné. Les rares visions cosmiques des Vitae sont à comprendre dans ce contexte : après l'expérience de Benoît d'un « monde... condensé en un unique rayon de soleil», le saint ne voit pas tant le monde physique rassemblé sous son regard que l'éternité des temps en Dieu (ex. 4). La vision du monde clos est ainsi décrite dans la Vie d'Alcuin (BHL 242) comme une expérience typologique de coïncidence entre le prophétisé et l'accompli.

Les préfaces en histoire sainte évoluent aux $\mathrm{IX}^{\mathrm{e}}-\mathrm{x}^{\mathrm{e}}$ siècles vers une lecture géographique de l'économie divine, indissociable d'un contexte d'évangélisation par les empires carolingien et ottonien (ex. 5 et 6). Membre d'une Église destinée à croître sur terre par les sacrements, les saints parcourent un monde harmonisé, occupé et habitable. Le monde se clôt, c'est-à-dire se parfait, en même temps qu'il est évangélisé donc inventorié voire cartographié. Dès le $\mathrm{xI}^{\mathrm{e}}$ siècle, la préface cosmique statique redevient majoritaire. 


\section{Modéliser le monde}

Les mondes clos peuvent constituer des espaces directement accessibles à la corporalité humaine. L'homme, microcosme au sein d'un microcosme, peut évoluer et prendre place dans un monde modélisé à son échelle.

Agnès du Vachat rappelle que le jardin, lieu réel ayant la capacité de juxtaposer plusieurs espaces normalement incompatibles ${ }^{16}$, peut fonctionner suivant trois modalités d'organisation et de rapport au monde.

La première est la clôture, ce que montre notamment l'Alhambra de Grenade (xIv ${ }^{e}$ siècle), organisé en une suite de cours et de jardinets clos. Sur la colline face à l'Alhambra se trouve le jardin du Généralife (xIV ${ }^{e}$ siècle), dont les terrasses successives comportent chacune un patio. Ici, si l'espace dévoué aux jardins diminue à mesure que l'on s'élève, la vue s'étend : bien que fondé sur la clôture, le jardin de l'Alhambra n'est pas un jardin de réclusion.

31 Ce principe d'ouverture visuelle - qui est la deuxième modalité - malgré la clôture est perceptible dans le premier patio du Généralife - patio de la Acequia -, qui comprend une galerie percée de jours (fig. 3).

Fig. 3. Alhambra, Jardin du Généralife, Patio de la Acequia (cl. A. du Vachat).

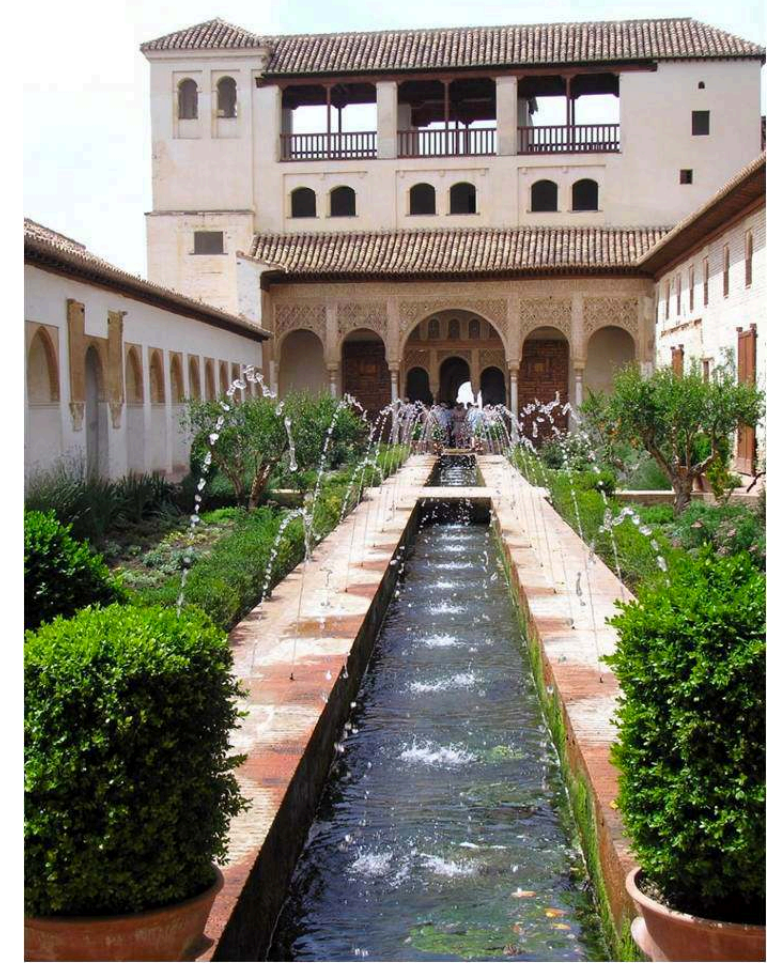

La vue peut ici se porter vers l'extérieur, offrant au regard la ville de Grenade et sa plaine cultivée comme une image de la relation entre la nature agricole, la «seconde nature » - Ciceron, De natura deorum -, et le jardin, la " troisième nature ", transformée à des fins d'agrément - Jacopo Bonfadio, Le Lettere, 1541. Les liens entre les deuxième et troisième natures sont visuels, mais aussi concrétisés par un système de canalisations. 

humaine. La compartimentation des jardins héritée de la Perse jouait un rôle capital. À Pasargades, le « paradis » construit par l'empereur Cyrus le Grand était un vaste parc de chasse. Clos de murs et situé au milieu des montagnes, il témoignait du pouvoir de Cyrus. Un tel aménagement était rendu possible par l'emploi de techniques hydrauliques élaborées qui, en irriguant l'ensemble du jardin, traversait ses différents compartiments pour structurer et ordonner son tracé.

La clôture est donc un dispositif tout à fait opérant pour lire les jardins du monde arabe, mais aussi latin, et ce jusqu'au XIXe siècle. L'intervenante propose ainsi une autre façon de miniaturiser l'espace qui l'érige en cosmos interne, comme un monde modélisé par sa clôture, mais aussi tourné vers l'infini par son ouverture sur le monde extérieur.

Alexandra Sotirakis conclut la journée en présentant la basilique Sant'Abbondio de Côme (1050-1060), fondée au $\mathrm{v}^{\mathrm{e}}$ siècle et devenue monastère bénédictin en 1010 pour la conservation des reliques de saint Abbondio (fig. 4).

Fig. 4. Abside de l'église Sant-Abondio (cl. A. Sotirakis).

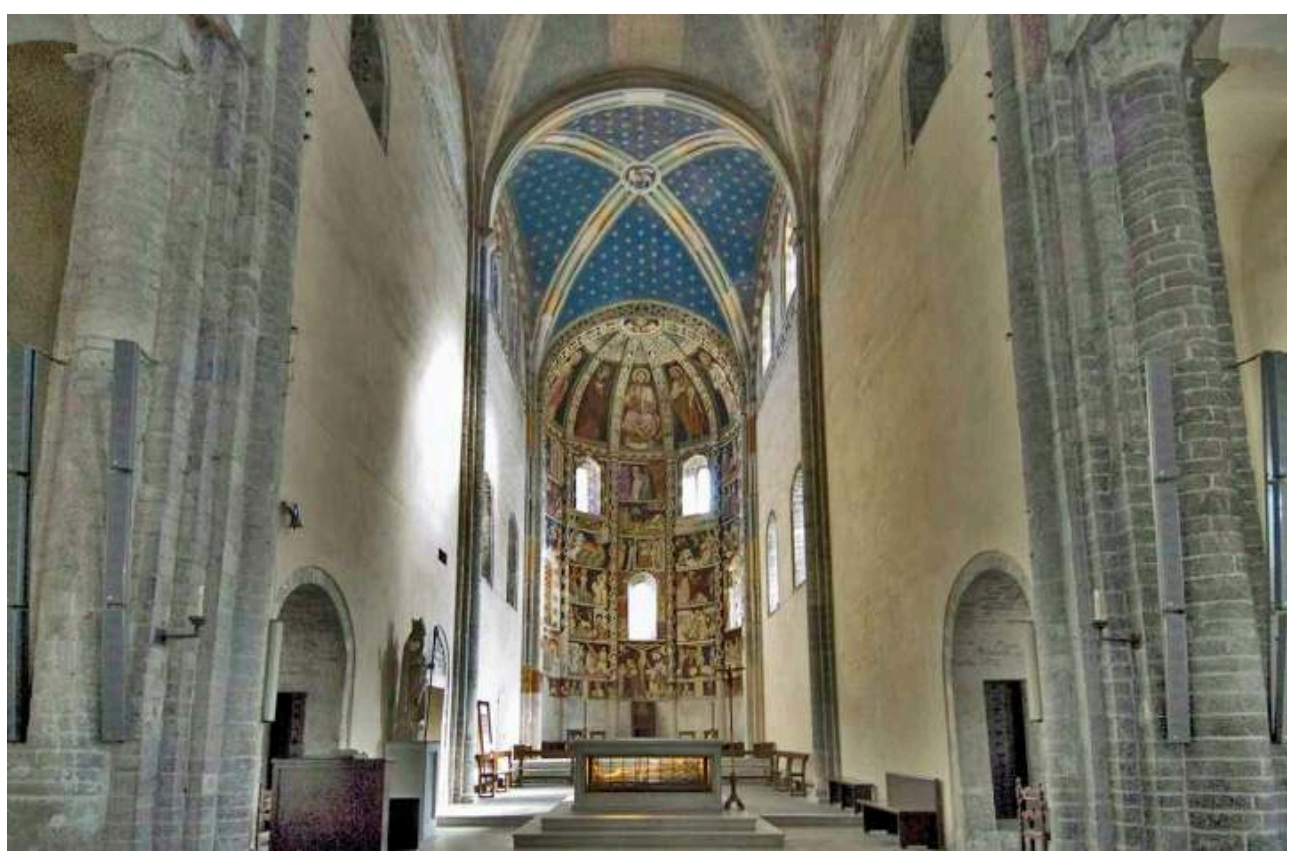

L'église est emblématique du diocèse de Côme et de sa situation singulière dans la géographie ecclésiastique: l'Église de Côme s'était en effet soustraite à l'autorité métropolitaine de Milan et était restée rattachée à la métropole d'Aquilée jusqu'à l'époque moderne, en raison de sa fidélité à la doctrine monophysite des Trois Chapitres défendue par Abbondio, mais condamnée par Justinien dès le milieu du $\mathrm{VI}^{\mathrm{e}}$ siècle. Les solutions architecturales conçues pour cette église dans la seconde moitié $\mathrm{du} \mathrm{XI}{ }^{\mathrm{e}}$ siècle semblent entrer dans la tradition comasque et s'imposer comme des signes d'identité locale ${ }^{17}$.

La plus remarquable de ces solutions est l'attention portée au voûtement de pierre dans la partie orientale de l'édifice, alors que l'église de plan basilical est pour le reste couverte d'une charpente. Au-delà de motivations techniques ou acoustiques, qui ne s'avèrent pas entièrement satisfaisantes, ce choix d'un couvrement de pierre se 
comprend davantage lorsqu'il est mis en perspective avec d'autres moyens structurels et décoratifs spécifiques à cette partie de l'édifice : le changement de niveau des sols, la densité du décor sculpté et la présence d'une barrière de chœur participent tous à mettre en valeur et à cloisonner les espaces orientaux. Tous ces éléments conduisent à instaurer une partition progressive de l'édifice sur son axe ouest-est et sont autant de marqueurs des différents seuils que doit franchir le fidèle ou le célébrant vers l'espace le plus sacré, l'autel.

38 L'intervenante invite également à mettre en relation l'appareil de pierre couvrant l'autel de l'église avec l'image théologique des pierres vivantes (les chrétiens) réunies autour de la pierre angulaire (le Christ-autel) pour constituer l'Église (l'assemblée), tirée du Nouveau Testament (1 Pi 2, 5) et devenue topos de l'exégèse. La spatialisation des volumes architecturaux participe ainsi à cloisonner et à ordonner l'assemblée des chrétiens réunis dans l'édifice.

Les particularités architecturales de Sant'Abbondio de Côme s'affirment comme un témoin local, proprement comasque, d'une dynamique spatiale concevant l'église comme un lieu entier et fini, un monde en soi, édifice communautaire dans lequel sont hiérarchisés les membres de l'Église comasque.

\section{Conclusion}

Durant cette première rencontre articulée sur un dialogue entre l'objet et sa clôture, le concept de clôture est apparu plus opérant que celui d'objet, surtout vu comme un ensemble à unité de sens. L'idée que la clôture soit à la fois garante de l'intégrité et de la mise en ordre a été validée, bien que ces deux effets doivent désormais être affinés, déclinés et historicisés.

41 Parmi les apports de la journée, l'idée d'infini a clivé l'interrogation originelle, au moment de comparer la pensée d'Hugues de Saint-Victor, pour qui la Loi d'un monde insaisissable peut être approchée par une logique herméneutique, et celle d'Albert le Grand pour qui l'infini, incommensurable, nécessite l'abstraction mathématique pour approcher sa logique.

L'idée d'occupation est apparue de façon inattendue et polysémique. Occuper le monde, l'habiter, le peupler ou le sanctifier sont autant de manières de superposer la perception humaine du monde et l'abstraction de son étendue totale, organisée par diverses dynamiques, géographiques, historiques et sacrées.

43 L'occupation a ainsi souvent rejoint la question du point de vue. C'est à partir du ciel astronomique, architectural ou spirituel - que le monde est contemplé, envisagé, voire saisi. En retour, le ciel participe, dans certains cas, à l'ordonnancement des lieux sur terre : la voûte du monument, le plafond peint, les régions célestes distribuées sur terre à travers les saints, non sans rappeler la pratique antique de l'arpentage.

Enfin, le thème de l'irrigation est constamment revenu: l'eau vivifiante de la mappemonde ou du jardin, la lumière illuminant l'édifice ou portant la vie par l'évangélisation, ou encore les peintures d'un plafond agencées sur la trajectoire de l'axe sacré de son église.

45 Parmi les perspectives de nos futurs travaux, l'une consisterait à historiciser mieux encore le fait que nous tentons de cerner. Une autre serait de scruter la complémentarité des deux dynamiques organisationnelles qui ont émergé de la 
journée, la centralisation et la polarisation. De la centralisation dépend une « concentricité » - à partir de la source, du spiritus, de l'autel, du sacrement, etc. -, qu'un phénomène de polarisation, au sens d'une dynamique entre deux extrémités en tension, est venu compléter - par exemple l'axe sacré d'une église modélisant la dynamique historique est-ouest de l'évangélisation. L'insertion de la clôture dans ce couple de dynamiques pourrait apporter des pistes de compréhension au paradoxe de la contention et de l'expansion à l'intérieur d'un monde clos. Ces dynamiques posent enfin la question de la modularité pour la compréhension et la reproduction du monde.

\section{Programme}

- Emmanuelle Vagnon (CNRS, LaMOP, UMR 8589) : «L'interprétation de l'image du monde, d'Isidore de Séville à Hugues de Saint-Victor. La mappemonde circulaire du manuscrit de Munich (Bayerische Staatsbibliothek, clm 10058, fol. 154vº)»

- Thomas Le Gouge (doctorant, université Paris 1 Panthéon-Sorbonne) : «L'œil géométrique du De causis d'Albert le Grand »

- Maud Pérez-Simon (IUF, Sorbonne Nouvelle-Paris 3) : «Un monde au plafond »

- Marie-Céline Isaïa (IUF, université Jean-Moulin Lyon 3, UMR 5648 Ciham) : «Jusqu'aux limites du monde. L'univers comme monde clos dans l'hagiographie latine (IV ${ }^{\mathrm{e}}-\mathrm{XI}^{\mathrm{e}}$ siècle) »

- Agnès du Vachat (École nationale supérieure de Paysage, Versailles) : «Enclore le monde dans un jardin, ouvrir le jardin sur le paysage : l'exemple des jardins de l'Alhambra »

- Alexandra Sotirakis (docteure, Centre André Chastel, Paris Sorbonne) : «Le monde dans l'église et l'église dans le monde. Sant'Abbondio de Côme, espace rituel et espace architectural »

Reçu : 31 août 2020 - Accepté : 16 octobre 2020

\section{NOTES}

1. M. BEAUd, L. CARTERon, E. ROUQUeTte et K. MitAlaïTE, « Journée d'étude : L'objet monde au Moyen Âge » dans Les carnets du LaMOP, en ligne [https://lamop.hypotheses.org/6400] mis en ligne le 17/01/2020, consulté le 01/07/2020.

2. MARIUS VICTORINUS, Liber de difinitionibus, éd. PL, 64, col. 907, expression longtemps attribuée à Boèce : R. FINCKH, Minor mundus homo. Studien zur Mikrokosmos-Idee in der mittelalterlichen Literatur, Göttingen, 1999, p. 53, n. 136.

3. ISIDORE DE SÉVILLE, De Natura rerum, IX, éd. PL, 83, col. 978 ; BÈDE LE VÉNÉRABLE, De Temporum ratione, XXXV, éd. PL, 90, col. 458.

4. BOĖCE, De Musica, I, 2, éd. PL, 63.

5. ISIDORE DE SÉVILLE, De Natura rerum, IX, 2, éd. CPL, 1188.

6. THÉODULF D'ORLÉANS, poème XLVI, in Theodulfi Carmina, éd. E. DÜMMLER, MGH, Poetarum Latinorum Medii Aeui, t. 1, Berlin, 1881, p. 544.

7. E. VAGNON, Exemplier, 10/02/2020, archive en ligne [https://hdl.handle.net/11280/5b60f072], mis en ligne le $01 / 09 / 2020$. 
8. P. GAUTIER DAlChÉ, La «Descriptio mappe mundi» de Hugues de Saint-Victor. Texte inédit avec introduction et commentaire, Turnhout, 1988.

9. ALBERT LE GRAND, De causis proprietatum elementorum, éd. P. HOSSFELD, ALBERTUS MAGNUS, Opera omnia, t. 5, vol. 2, Aschendorff, 1980, p. 49-104.

10. T. LE GOUGE, Exemplier, 10/02/2020, archive en ligne [https://hdl.handle.net/11280/08e41352], mis en ligne le 01/09/2020.

11. JOANNES DE SACRO BOSCO, The Sphere of Sacrobosco and its commentators, éd. L. THORNDIKE, Chicago, 1949.

12. WIEN, Österreichische Nationalbibliothek, cod. 273.

13. D. GRENET, « Exposition Charpentes et plafonds peints du Moyen Âge, Paris, 6 février- 6 mars 2020 », en ligne [http://rcppm.org/blog/2020/01/exposition-charpentes-et-plafonds-peints-du-moyenage-paris-6-fevrier-6-mars-2020], mis en ligne le 30/01/2020, consulté le 31/08/2020.

14. G. DE FRANCISCO, Il soffitto dipinto della Cattedrale di Nicosia, Enna, 1997.

15. M.-C. ISAÏA, Exemplier, 10/02/2020, archive en ligne [https://hdl.handle.net/11280/9f8c5adc], mis en ligne le 01/09/2020.

16. M. FOUCAULT, « Des espaces autres » [1967], in Dits et écrits 1954-1988, t. 4, Paris, 1994, p. 759.

17. A. SotIRAKIS, Espaces voûtés du chevet dans l'architecture romane en Europe: formes et fonctions, thèse de doctorat, Sorbonne université, 2020.

\section{AUTEURS}

\section{MATHIEU BEAUD}

INHA

\section{LUCE CARTERON}

CERAM, université Paris 3

\section{KRISTINA MITALAÏTE}

LEM, CNRS

\section{ENIMIE ROUQUETTE}

CERAM, université Paris 3 